\title{
Mulan: a part-per-million measurement of the muon lifetime and determination of the Fermi constant
}

\author{
Robert Carey $^{1}$, Tim Gorringe ${ }^{2 \star}$ and David W. Hertzog ${ }^{3}$
}

1 Dept. of Physics, Boston University, Boston, MA 02215, USA

2 Dept. of Physics and Astronomy, University of Kentucky, Lexington, KY 40506, USA

3 Dept. of Physics, University of Washington, Seattle, WA 98195, USA

$$
\star \text { tim.gorringe@uky.edu }
$$

PAUL SCHERRER INSTITUT

\begin{abstract}
The part-per-million measurement of the positive muon lifetime and determination of the Fermi constant by the MuLan experiment at the Paul Scherrer Institute is reviewed. The experiment used an innovative, time-structured, surface muon beam and a near- $4 \pi$, finely-segmented, plastic scintillator positron detector. Two in-vacuum muon stopping targets were used: a ferromagnetic foil with a large internal magnetic field, and a quartz crystal in a moderate external magnetic field. The experiment acquired a dataset of $1.6 \times 10^{12}$ positive muon decays and obtained a muon lifetime $\tau_{\mu}=2196980.3(2.2) \mathrm{ps}$ $(1.0 \mathrm{ppm})$ and Fermi constant $\mathrm{G}_{F}=1.1663787(6) \times 10^{-5} \mathrm{GeV}^{-2}(0.5 \mathrm{ppm})$. The thirty-fold improvement in $\tau_{\mu}$ has proven valuable for precision measurements in nuclear muon capture and the commensurate improvement in $\mathrm{G}_{F}$ has proven valuable for precision tests of the standard model.
\end{abstract}

(c) (1) Copyright R. Carey et al.

This work is licensed under the Creative Commons Attribution 4.0 International License.

Published by the SciPost Foundation.
Received 09-02-2021

Accepted 01-07-2021

Published 06-09-2021

doi:10.21468/SciPostPhysProc.5.016

\subsection{Introduction}

The electromagnetic $\left(\alpha_{e}\right)$, strong $\left(\alpha_{s}\right)$, gravitational $(G)$ and weak $\left(G_{F}\right)$ couplings are the "calibration constants" of nature [1]. Their magnitudes haven't been determined by theory but rather are obtained from measurement. Collectively, they determine the dynamics and bindings of microscopic and macroscopic matter and the character of the universe.

The fine-structure constant $\alpha_{e}$ governs the scale of atomic energy levels and the rates of all electromagnetic processes. It is known to the astonishing precision of 0.15 parts per billion.

The energy-scale-dependent effective coupling $\alpha_{s}$ governs the binding of protons and neutrons to form nuclei and the production of chemical elements in stars. It also controls the emergence of the two faces of the strong interaction: quark confinement at large distances and asymptotic freedom at short distances. 
Despite the omnipresence of the gravitational force and its implications for the structure of the universe, the precision determination of the gravitational constant $\mathrm{G}$ has been deceptively difficult. Since its original measurement by Cavendish, the surprising inconsistences between modern methods have meant little overall improvement in our knowledge of this constant [2].

Finally, the weak interaction governs the thermonuclear reactions in the sun that are ultimately responsible for light, energy and life. The understanding of weak interactions enables the computation of phenomena from cosmology and astrophysics to nuclear and particle physics, including exacting tests of electroweak theory. Fermi described the weak processes by a simple four-fermion contact interaction with the coupling strength that became known as $G_{F}$. This constant and the current-current weak interaction description have survived many decades as a very convenient, low-energy, effective theory. Of course, our modern understanding of weak interactions has evolved to incorporate such features as parity violating $V-A$ currents and heavy $W, Z$ gauge bosons, in a unified electroweak theory described by two gauge couplings and the Higgs energy density. The Fermi constant $G_{F}$, together with measurements of $\alpha_{e}$ and $M_{Z}$, provide by far the best determinations of the gauge couplings and Higgs energy density.

Since its discovery in 1933, the muon, a heavy sibling of ordinary electrons, has played a significant role in subatomic physics. Muons are undoubtedly the best tool for the precise determination of the Fermi constant and, uniquely from the considerations above, provide by far the most precise measure of the weak coupling. From the theoretical perspective, the purely-leptonic muon decay is well suited to precision calculations within the Fermi theory, and from the experimental perspective, its microsecond-scale lifetime is well suited to modern techniques for time measurements. Because the best method to determine $G_{F}$ is from the muon lifetime, it is appropriate to recognize that what is measured is $G_{\mu}$, the muon constant in weak decay. The assumption of lepton universality allows the relation $G_{F} \equiv G_{\mu}$, which we assume here, but can and should be challenged by other weak interaction processes.

An important breakthrough for determining $\mathrm{G}_{F}$ was work by van Ritbergen and Stuart [3] and Pak and Czarnecki [4]. Using Fermi theory with 2-loop QED corrections, these authors reduced the theoretical uncertainty in the relation between the muon lifetime and the Fermi constant from 15 parts-per-million to 150 parts-per-billion. Their work thus opened the door for the MuLan experiment at PSI [5,6], a part-per-million measurement of the muon lifetime $\tau_{\mu}$ and determination of the Fermi constant $\mathrm{G}_{F}-$ a thirty-fold improvement over earlier measurements.

\subsection{Experimental setup}

The principle of the MuLan measurement of the muon lifetime is straightforward.

First, prepare a small "source" of positive muons. Next, measure the times of decay positrons. Finally, construct the exponential decay curve and extract the positive muon lifetime. In practice we repeated the sequence of source preparation and positron measurement at approximately $30 \mathrm{KHz}$ over a period of roughly 20 weeks in two running periods.

The experiment used longitudinally polarized, $29 \mathrm{MeV} / \mathrm{c}$, positive muons from the $\pi \mathrm{E} 3$ secondary beamline at the PSI proton cyclotron. Incoming muons were stopped in solid targets and outgoing positrons were detected in a near- $4 \pi$, finely segmented, fast-timing, plastic scintillator positron detector. The analog signals from individual scintillators were recorded by 450 MSPS (mega samples per second) waveform digitizers and accumulated by a high-speed data acquisition system.

One innovative feature of the system was the imposition of time structure in the $\pi \mathrm{E} 3$ beamline. The experiment operated in repeating cycles of beam-on accumulation periods, in which surface muons were accumulated in the stopping target, and beam-off measurement periods, in which decay positrons were detected in the MuLan detector. The time structure 
avoided the need to associate the decay positrons with parent muons - a limiting factor of earlier experiments using continuous beams.

The specific time structure comprised a $5 \mu$ s-long beam-on accumulation period $\left(T_{A}\right)$, and a $22 \mu$ s-long beam-off measurement period $\left(T_{M}\right)$. The time structure was imposed on the $\pi \mathrm{E} 3$ beam using a custom-built, fast-switching, $25 \mathrm{kV}$ electrostatic kicker. When the kicker was de-energized, the muons were transported to the Target; when the kicker was energized, the muons were deflected into a collimator. A sample time distribution of incoming muons and outgoing positrons that illustrates the accumulation and measurement periods is shown in Figure 16.1.

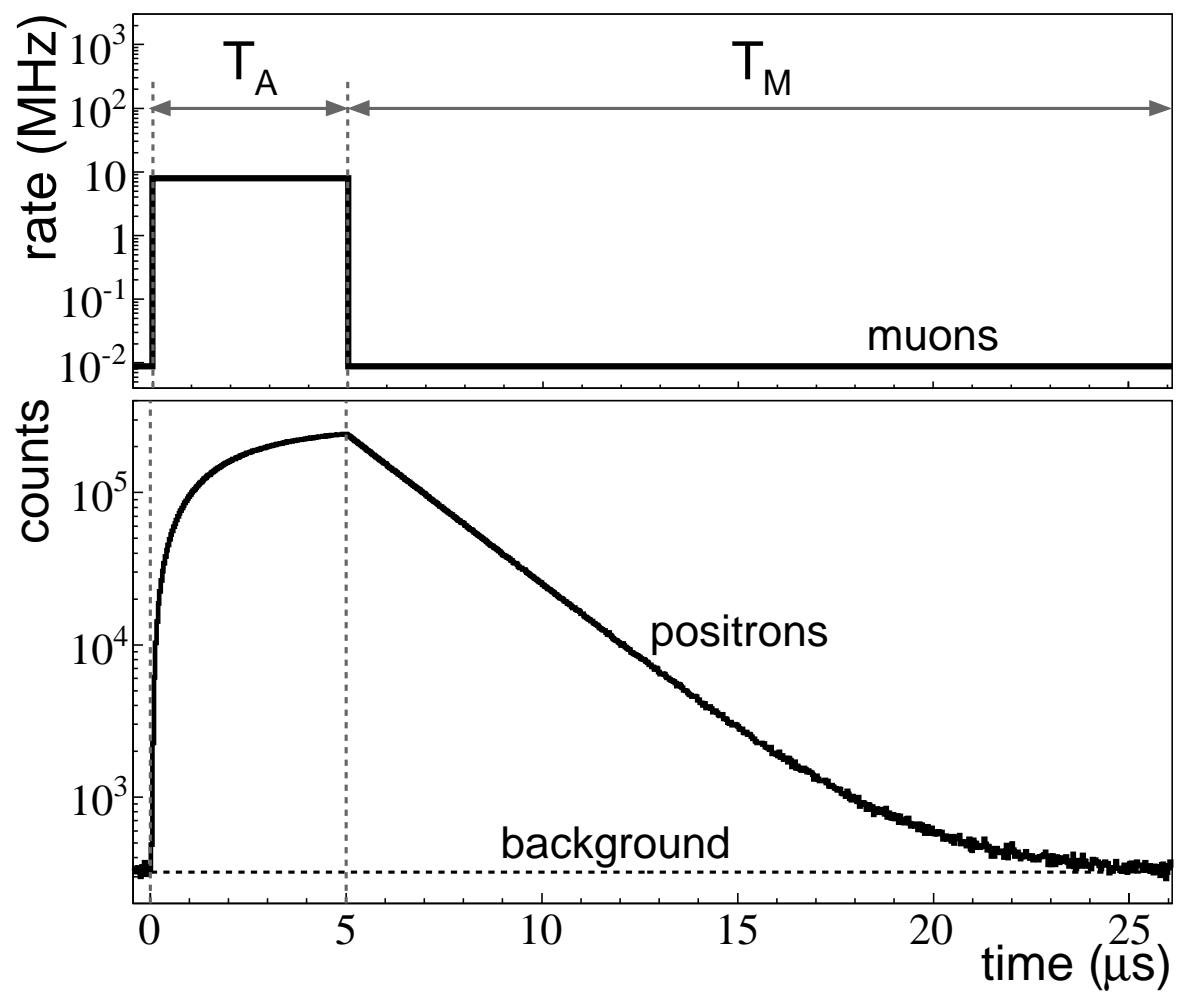

Figure 16.1: Plot of the time dependence of the muon arrival rate (upper panel) and decay positron counts (lower panel) that was imposed by the electrostatic kicker. The durations of the beam-on accumulation period and beam-off measurement period were $T_{A}=5 \mu \mathrm{s}$ and $T_{M}=22 \mu \mathrm{s}$, respectively. Figure courtesy of the MuLan collaboration.

Other innovative features of the experiment were the use of in-vacuum stopping targets and near- $4 \pi$ positron detection. A consequence of parity violation in weak interactions is that the emitted positrons in muon decay are asymmetrically distributed about the muon spin direction. This poses a problem as spin precession and spin relaxation of stopped muons could distort the pure exponential time distribution of the decay positrons and bias the extraction of $\tau_{\mu}$.

A fully $4 \pi$, perfectly isotropic, positron detector would negate this issue of precession and relaxation by detecting positrons with identical probability in all directions. The Mulan combination of an in-vacuum, detector-centered target for incoming muons and near- $4 \pi$, nearisotropic, detector for outgoing positrons, was an important part of the experimental strategy for minimizing such spin precession and relaxation effects.

In addition, the experiment deployed two different combinations of stopping target materi- 


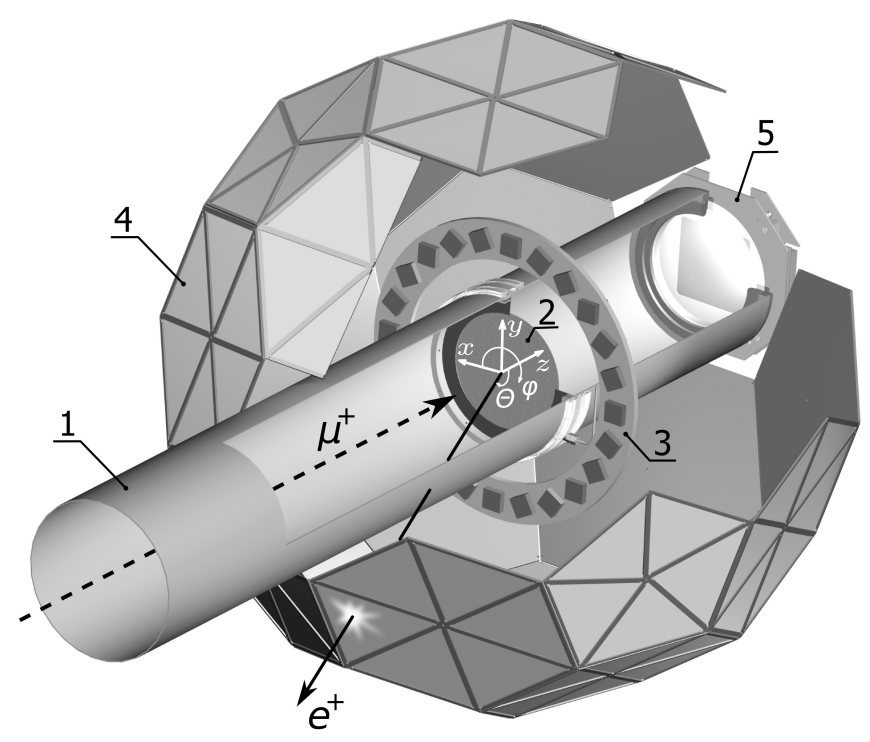

Figure 16.2: A cutaway view of the Mulan experimental setup showing the (1) vacuum beamline, (2) in-vacuum stopping target, (3) Halbach arrangement permanent magnet, (4) soccer ball geometry scintillator array, and (5) beam monitor. We used the Halbach magnet for the external magnetic field in the quartz target data-taking. Figure courtesy of the MuLan collaboration.

als and transverse magnetic fields in order to further reduce the spin precession and relaxation effects. One setup involved a magnetized Fe-Cr-Co foil (Arnokrome-III) with a $\sim 4 \mathrm{kG}$ internal $B$-field. Another setup involved a quartz crystal disk $\left(\mathrm{SiO}_{2}\right)$ in a $130 \mathrm{G}$ external $B$-field. In the ferromagnetic target, where muons reside as diamagnetic ions, the $\mu^{+}$precession frequency was about $50 \mathrm{MHz}$. In the quartz target the primary muonium $\left(\mu^{+} e^{-}\right)$population has a $180 \mathrm{MHz}$ precession frequency and the secondary muon $\left(\mu^{+}\right)$population has a $1.8 \mathrm{MHz}$ precession frequency. In both strategies, spin dephasing during muon accumulation yielded a roughly 1000 -fold reduction in the ensemble-averaged $\mu^{+}$polarization at the beginning of the measurement period.

The positron detector was constructed of 170 triangle-shaped, plastic scintillator pairs arranged in a soccer ball geometry (Figure 16.2). Each pair comprised an inner and outer scintillator tile. The pairs were grouped into ten pentagonal enclosures containing five tile-pairs and twenty hexagonal enclosures containing six tile-pairs, which together formed the soccer ball geometry. The segmentation was important in reducing positron pile-up in individual detector elements. The symmetric arrangement of detector elements was important in reducing the effects of muon spin rotation / relaxation.

\subsection{Data analysis}

A total of $1.1 \times 10^{12}$ decays from positive muon stops in Arnokrome-III and $5.4 \times 10^{11}$ decays from positive muon stops in quartz were collected. Other datasets with different orientations of the magnetic field and different centering of the muon stopping distribution were collected in order to study the systematic errors associated with spin precession and relaxation.

The time and amplitude of individual pulses were determined from least square fits to digitized waveform templates. The procedure fit a higher-resolution template waveform $(0.22 \mathrm{~ns}$ sampling-interval) to the lower-resolution individual waveforms (2.2 ns sampling-interval). The higher-resolution templates were constructed by combining a large sample of $2.2 \mathrm{~ns}$ 
sampling-interval, single positron, digitized waveforms. The fitting procedure would add / remove pulses to obtain the best $\chi^{2}$.

Positrons were defined as inner-outer tile-pair coincidences. In identifying the coincidences, cuts were applied to define an unambiguous amplitude threshold $A_{t h r}$ for detector hits and to define an unambiguous artificial deadtime (ADT) between detector pulses. Hits that survived these cuts were sorted into time distributions of inner singles, outer singles and inner-outer coincidences. The construction of coincidence histograms with different thresholds and deadtimes was important for studying the distortions that arise from pulse pileup and gain changes. The typical rates were 40 stopped muons per accumulation period and 15 detected positrons per measurement period. The nominal $13.3 \mathrm{~ns}$ ADT yielded a pileup distortion of roughly $10^{-3}$ at the start of the measurement period and roughly $10^{-7}$ at the end of the measurement period.

A hit is lost if it occurs in the artificial deadtime of an earlier hit. Our procedure for correcting for pileup took advantage of the time structure of the incident beam. The pileup losses were statistically recovered by replacing the lost hits in each measurement period with measured hits at equivalent times in neighboring measurement periods. For example, to correct for leading-order pileup, if a hit is observed at time $t_{i}$ in fill $j$ (the "trigger" hit), a hit is searched for within the interval $t_{i} \rightarrow t_{i}+\mathrm{ADT}$ in fill $j+1$ (the "shadow" hit). Adding the resulting histogram of shadow hit times to the original histogram of trigger hit times thus statistically recovers the lost hits (similar shadow methods were employed for handling the higher-order pileup).

As mentioned, only hits with amplitudes exceeding the threshold $A_{t h r}$ were used. Consequently, if the detector gain changes over the measurement period, then the time histogram will be distorted by either additional hits climbing above $A_{t h r}$ or additional hits falling below $A_{t h r}$ cut. We corrected for gain changes versus measurement time by monitoring changes in the positron minimally ionizing particle (MIP) peak amplitude over the measurement period.

A simple procedure was used to extract the lifetime $\tau_{\mu}$ from the Arnokrome-III target. The summed tile-pair time histogram of coincidence hits was fit to $N e^{-t / \tau_{\mu}}+C$. The approach relied on sufficient cancellation of Arnokrome-III precession and relaxation effects by combination of the spin dephasing and the opposite-pair detector geometry.

A more complicated procedure was needed to extract the lifetime $\tau_{\mu}$ from the quartz target. First, 170 geometry-dependent effective lifetimes were extracted for each tile-pair from fits to

$$
N(t)=N e^{-t / \tau_{\text {eff }}}[1+f(t)]+C,
$$

where $f(t)$ accounts for time-dependent effects of transverse-field (TF) spin precession and relaxation. Then, the true positive muon lifetime $\tau_{\mu}$ was extracted by fitting the effective lifetimes, $\tau_{\text {eff }}$, to

$$
\tau_{\text {eff }}\left(\theta_{B}, \phi_{B}\right)=\tau_{\mu}\left[1+\delta\left(\theta_{B}, \phi_{B}\right)\right],
$$

where $\delta\left(\theta_{B}, \phi_{B}\right)$ accounts for geometry-dependent effects of longitudinal-field (LF) spin relaxation. Together the two steps were sufficient to handle the effects of precession and relaxation in quartz.

\subsection{Results}

The individual results from the Arnokrome-III dataset and the quartz dataset, and the weighted average are given in Table 16.1.

The weighted average corresponds to an overall uncertainty in the positive muon lifetime of $2.2 \mathrm{ps}$, or $1.0 \mathrm{ppm}$. The largest contributions to the systematic uncertainties are associated with the aforementioned pulse pileup, gain changes, and muon precession and relaxation effects, as well as the knowledge of the time independence of the beam extinction during the 
Table 16.1: Muon lifetime results from the Arnokrome-III dataset, quartz dataset, and their weighted average.

\begin{tabular}{|l|c|}
\hline Target material & Positive muon lifetime (ps) \\
\hline Arnokrome-III & $2196979.9 \pm 2.5($ stat $) \pm 0.9($ syst $)$ \\
Quartz & $2196981.2 \pm 3.7($ stat $) \pm 0.9($ syst $)$ \\
\hline Weighted average & $2196980.3 \pm 2.1($ stat $) \pm 0.7$ (syst) \\
\hline
\end{tabular}

measurement period. The final result for $\tau_{\mu}$ is in agreement with the earlier work of Giovanetti et al. [7], Balandin et al. [8] and Bardin et al. [9].

We note the precision determination of $\tau_{\mu}$ is important to work on nuclear muon capture. The MuCap experiment [10] at PSI determined the $\mu$ p singlet capture rate from the small difference between the positive muon lifetime and the muonic hydrogen atom lifetime. Similarly, the MuSun experiment [11] at PSI will determine the $\mu \mathrm{d}$ doublet capture rate from the small difference between the positive muon lifetime and the muonic deuterium atom lifetime. These two experiments are described in Section 17 [12] and Section 18 [13], respectively.

The Fermi constant $G_{F}$ was extracted using the relation obtained by van Ritbergen and Stuart (vRS) [3] and yields $G_{F}($ MuLan $)=1.1663787(6) \times 10^{-5} \mathrm{GeV}^{-2}(0.5 \mathrm{ppm})$ - a thirtyfold improvement over the earlier 1998 Particle Data Group [14] value that pre-dated the vRS theoretical work and MuLan experimental work. The $0.5 \mathrm{ppm}$ error is dominated by the $1.0 \mathrm{ppm}$ uncertainty of the lifetime measurement, with contributions of $0.08 \mathrm{ppm}$ from the muon mass measurement and $0.15 \mathrm{ppm}$ from the theoretical corrections.

Together, the fine structure constant $\alpha$, Fermi coupling constant $\mathrm{G}_{F}$, and $\mathrm{Z}$ boson mass $\mathrm{M}_{Z}$, fix the electroweak parameters of the standard model. The thirty-fold improvement in the determination of the Fermi constant $\mathrm{G}_{F}$, together with other improvements in determinations of $\alpha$ and $\mathrm{M}_{Z}$, have allowed for improved tests of the standard model and improved searches for new phenomena.

We wish to thank our collaborators in the MuLan experiment and Paul Scherrer Institute for their exceptional organizational and technical support. We also wish to thank M. Barnes and G. Wait from TRIUMF for their development of the electrostatic kicker, Bill Marciano for advocating and promoting the experiment, and the U.S. National Science Foundation (NSF 1807266) and U.S. Department of Energy (DOE DE-FG02-97ER41020) for their financial support.

\section{References}

[1] P. Zyla et al., Review of particle physics, Prog. Theor. Exper. Phys. 083C01 (2020), doi:10.1093/ptep/ptaa104.

[2] C. Rothleitner and S. Schlamminger, Measurements of the newtonian constant of gravitation, Rev. Sci. Instrum. 88, 111101 (2017), doi:10.1063/1.4994619.

[3] T. van Ritbergen and R. G. Stuart, On the precise determination of the Fermi coupling constant from the muon lifetime, Nucl.Phys. B 564, 343 (2000), doi:10.1016/S05503213(99)00572-6.

[4] A. Pak and A. Czarnecki, Mass effects in muon and semileptonic $b \rightarrow c$ decays, Phys. Rev. Lett. 100, 241807 (2008), doi:10.1103/PhysRevLett.100.241807. 
[5] D. Webber et al., Measurement of the positive muon lifetime and determination of the Fermi constant to part-per-million precision, Phys. Rev. Lett. 106, 041803 (2011), doi:10.1103/PhysRevLett.106.041803.

[6] V. Tishchenko et al., Detailed report of the MuLan measurement of the positive muon lifetime and determination of the Fermi constant, Phys. Rev. D 87, 052003 (2013), doi:10.1103/PhysRevD.87.052003.

[7] K. Giovanetti et al., Mean life of the positive muon, Phys. Rev. D 29, 343 (1984), doi:10.1103/PhysRevD.29.343.

[8] M. Balandin, V. Grebenyuk, V. Zinov, A. Konin and A. Ponomarev, Measurement of the lifetime of the positive muon, Sov. Phys. JETP 40, 811 (1975), Available at http://ivanik3. narod.ru/TimeLifeMezon/e_040_05_0811Balandin.pdf.

[9] G. Bardin, J. Duclos, A. Magnon, J. Martino, E. Zavattini, A. Bertin, M. Capponi, M. Piccinini and A. Vitale, A new measurement of the positive muon lifetime, Phys. Lett. B 137, 135 (1984), doi:10.1016/0370-2693(84)91121-3.

[10] V. Andreev et al., Measurement of the rate of muon capture in hydrogen gas and determination of the proton's pseudoscalar coupling $g_{P}$, Phys. Rev. Lett. 99, 032002 (2007), doi:10.1103/PhysRevLett.99.032002.

[11] P. Kammel, Precision muon capture at PSI, Proc. Sci. 172, 016 (2013), doi:10.22323/1.172.0016.

[12] M. Hildebrandt and C. Petitjean, MuCap: Muon capture on the proton, SciPost Phys. Proc. 5, 017 (2021), doi:10.21468/SciPostPhysProc.5.017.

[13] P. Kammel, MuSun - Muon capture on the deuteron, SciPost Phys. Proc. 5, 018 (2021), doi:10.21468/SciPostPhysProc.5.018.

[14] C. Caso et al., Review of particle physics. Particle data group, Eur. Phys. J. C 3, 1 (1998), doi:10.1007/s10052-998-0104-x. 